\title{
Criminologie
}

\section{Éditorial : la prévention de l'inadaptation sociale : approche développementale}

\section{Michel Jonasz}

Volume 31, numéro 1, printemps 1998

La prévention de l'inadaptation sociale : approche développementale

URI : https://id.erudit.org/iderudit/017408ar

DOI : https://doi.org/10.7202/017408ar

Aller au sommaire du numéro

Éditeur(s)

Les Presses de l'Université de Montréal

ISSN

0316-0041 (imprimé)

1492-1367 (numérique)

Découvrir la revue

Citer ce document

Jonasz, M. (1998). Éditorial : la prévention de l'inadaptation sociale : approche développementale. Criminologie, 31(1), 3-5. https://doi.org/10.7202/017408ar d'utilisation que vous pouvez consulter en ligne.

https://apropos.erudit.org/fr/usagers/politique-dutilisation/ 
La prévention de la criminalité adulte suit de multiples chemins. Approches répressives et dissuasives, approches situationnelles et matérielles, interventions communautaires et sociopolitiques, voilà autant d'avenues empruntées pour limiter l'étendue de l'activité délictueuse. À cette liste, non exhaustive, s'ajoute l'approche développementale. Dans l'ensemble, les tenants de cette perspective conçoivent les manifestations de l'inadaptation comme la résultante de l'interaction entre l'individu, avec ses caractéristiques biologiques et psychologiques, et son environnement social, particulièrement la famille, le groupe de pairs et l'école. Les signes d'inadaptation apparaissent lorsque l'individu peine à répondre aux exigences normatives de son environnement social, et lorsque ce dernier ne répond pas adéquatement à ses besoins. Chaque étape du développement (petite enfance, enfance, adolescence) appelle la réalisation de tâches développementales qui permettent d'accroître la capacité d'adaptation des individus, de développer les habiletés nécessaires pour remplir les tâches de l'étape suivante. Plus les problèmes d'appariement entre l'individu et son environnement se produisent tôt dans le développement, plus graves sont les conséquences à long terme puisqu'elles affectent les capacités de l'individu et de son environnement à répondre aux exigences développementales ultérieures. La recherche sur l'étiologie de l'inadaptation psychosociale des enfants et des adolescents a permis de mettre en lumière l'impact dramatique de certaines caractéristiques individuelles, innées ou acquises (p. ex., intelligence, habiletés sociocognitives et sociales, agressivité et contrôle des émotions, etc.) et de certains processus sociaux (p. ex., attachement et pratiques éducatives familiales déficientes, rejet social et association à des pairs déviants, échec scolaire et exclusion socioprofessionnelle) (Loeber et Farrington, 1998 ; Vitaro, Dobkin, Gagnon et Le Blanc, 1994). Ces caractéristiques individuelles et sociales deviennent alors les cibles privilégiées de l'intervention préventive. Les textes choisis pour ce numéro

1. Professeur adjoint à l'École de psychoéducation de l'Université de Montréal et chercheur au Groupe de recherche sur les adolescents en difficulté (GRAD), au Centre de recherche et d'intervention sur la réussite éducative (CRIRES) et à l'Institut pour le développement social des jeunes (IDSJ). Université de Montréal, C.P. 6128, Succursale Centre-Ville, Montréal, (Québec), Canada, H3C 3J7. Adresse électronique : janoszm@ere.umontreal.ca 
illustrent différentes stratégies d'intervention préventive qui s'inscrivent à l'intérieur d'une approche développementale.

L'étude des trajectoires développementales menant à la délinquance, plus spécifiquement le cas des délinquants violents et persistants, a mis en lumière un facteur de risque précoce important, celui des mères adolescentes. Ces jeunes femmes, par leurs caractéristiques personnelles et sociales, risquent plus d'avoir des garçons agressifs qui seront, à leur tour, plus à risque de devenir délinquants. Une voie prometteuse de prévention consiste donc à aider ces jeunes mères à offrir un contexte éducatif qui minimise les risques d'un tel développement chez leur enfant. C'est cette problématique qui retient l'attention de Tarabulsy, Robitaille, Lacharité, Deslandes et Coderre, qui présentent les caractéristiques d'un programme de prévention auprès de mères adolescentes, programme qui repose sur le modèle théorique de l'attachement.

Haggerty, Catalano, Harachi et Abbott présentent quant à eux l'implantation d'une étude expérimentale de prévention dans dix écoles primaires de la banlieue de Seattle (côte ouest américaine). Version améliorée du Seattle Social Development Project (Hawkins, Catalano, Morrison, O'Donnell, Abbott et Day, 1992) qui a déjà démontré son efficacité à court et à moyen terme, ce projet de prévention primaire cherche à soutenir le développement de liens entre le jeune, sa famille et l'école. Les interventions mises en place visent à maximiser la qualité de l'enseignement et de l'encadrement en classe, la qualité des pratiques éducatives familiales, et la qualité des habiletés sociales des jeunes.

Vitaro et Tremblay explorent, quant à eux, l'effet d'une intervention précoce menée auprès de jeunes garçons agressifs québécois issus de milieux défavorisés et auprès de leur famille, sur l'association ultérieure à des pairs déviants. Les auteurs se demandent dans quelle mesure l'impact de leurs interventions sur les conduites délinquantes passe par une réduction de l'influence des pairs, un facteur précipitant bien documenté des difficultés comportementales à l'adolescencc.

Poulin, Dishion, Kavanagh et Kiesner, de l'Oregon Social Learning Center d'où proviennent les travaux de Gerald Patterson, cherchent à intervenir directement auprès des adolescents à risque et de leur famille. Les auteurs décrivent les caractéristiques et quelques données d'implantation de la seconde version du Adolescent Transition Program (ATP). La première version avait démontré son efficacité auprès des parents mais des effets iatrogéniques (négatifs) ont récemment été identifiés. Le regroupement d'adolescents à risque semble être à la base de cet effet non recherché. La nouvelle version du ATP tente de contourner le problème en se centrant sur les parents et en offrant un programme à entrées progressives intégré à l'école. 
Janosz, Le Blanc et Boulerice abordent le problème du décrochage scolaire. En étudiant l'impact de la consommation de psychotropes et des conduites délinquantes sur le décrochage, selon différents types de décrocheurs, les auteurs démontrent la nécessité d'adopter une approche différentielle dans la prévention du décrochage (Le Blanc, 1990).

Enfin, Hastings nous propose une analyse critique de la prévention du crime par le développement social, notion mise de l'avant par le Conseil national de prévention du crime. Il en profite pour comparer les forces et limites de trois approches en prévention : l'approche développementale, sociale et communautaire.

Le lecteur sera peut-être surpris de la disparition de la section « hors thème ». Cet aménagement est exceptionnel. Compte tenu du nombre d'articles retenus et de la place disponible, le comité de rédaction a voulu privilégier l'intégrité thématique du numéro.

\section{BIBLIOGRAPHIE}

HAWKINS, J.D., CATALANO, R.F., MORRISON, D.M., O'DONNELL, J., ABBOTT, R.D. et DAY, L.E. (1992), " The Seattle social development project : effects of the first four years on protective factors and problem behaviors " in McCORD, J. et TREMBLAY, R.E. (dir.), Preventing Antisocial Behavior -Interventions from Birth through Adolescence, (p. 139-161), The Guilford Press, New York.

LE BLANC, M. (1990), «L'intervenant auprès des jeunes délinquants : un omnipraticien ou un spécialiste ? " Revue canadienne de Psycho-Éducation, vol. 18, n² 2, p. 85-100.

LOEBER, R. et FARRINGTON, D.P. (1998), Serious and Violent Juvenile Offenders : Risk Factors and Successful Interventions, Thousands Oaks, Sage.

TREMBLAY, R.E., VITARO, F., BERTRAND, L., LE BLANC, M., BEAUCHESNE, H., BOILEAU, H. et DAVID, L. (1992), « Parent and child training to prevent early onset of delinquency: The Montréal longitudinal-experimental study " in McCORD, J. and TREMBLAY, R.E. (dir.), Preventing Antisocial Behavior - Interventions from Birth through Adolescence, (p. 117-138), The Guilford Press, New York.

VITARO, F., DOBKIN, P.L., GAGNON, C. et LE BLANC, M. (1994), Les problèmes d'adaptation psychosociale chez l'enfant et l'adolescent : prévalence, déterminants et prévention, Québec, Presses de l'Université du Québec. 